\title{
A Real p-Homogeneous Seminorm with Square Property Is Submultiplicative
}

\author{
Mohammed El Azhari \\ Department of Mathematics, Ecole Normale Supérieure, Rabat, Morocco \\ Email: mohammed.elazhari@yahoo.fr
}

Received October 10, 2013; revised November 10, 2013; accepted November 17, 2013

Copyright (C) 2013 Mohammed El Azhari. This is an open access article distributed under the Creative Commons Attribution License, which permits unrestricted use, distribution, and reproduction in any medium, provided the original work is properly cited.

\begin{abstract}
We give a functional representation theorem for a class of real p-Banach algebras. This theorem is used to show that every p-homogeneous seminorm with square property on a real associative algebra is submultiplicative.
\end{abstract}

Keywords: Functional Representation; p-Homogeneous Seminorm; Square Property; Submultiplicative

\section{Introduction}

J. Arhippainen [1] has obtained the following result:

Theorem 1 of [1]. Let $q$ be a p-homogeneous seminorm with square property on a complex associative algebra $\mathrm{A}$. Then

1) $\operatorname{Ker}(q)$ is an ideal of $A$;

2) The quotient algebra $A / \operatorname{Ker}(q)$ is commutative;

3) $q$ is submultiplicative;

4) $q^{\frac{1}{p}}$ is a submultiplicative seminorm on $A$.

This result is a positive answer to a problem posed in [2] and considered in [3-5]. The proofs of (3) and (4) depend on (2) which is obtained by using a locally bounded version of the Hirschfeld-Zelazko Theorem [1, Lemma 1]. This method can not be used in a real algebra; if $\mathrm{q}$ is the usual norm defined on the real algebra $\mathrm{H}$ of quaternions, $\operatorname{Ker}(q)=\{0\}$ and $\mathrm{H} / \operatorname{Ker}(q) \cong \mathrm{H}$ is noncommutative, then the assertion (2) does not hold in the real case.

The purpose of this paper is to provide a real algebra analogue of the above Arhippainen Theorem, and this improves the result in [6]. Our method is based on a functional representation theorem which we will establish; it is an extension of the Abel-Jarosz Theorem [7, Theorem 1] to real p-Banach algebras. We also give a functional representation theorem for a class of complex p-Banach algebras. As a consequence, we obtain the main result in [8].

\section{Preliminaries}

Let $\mathrm{A}$ be an associative algebra over the field $\mathrm{K}=\mathrm{R}$ or $\mathrm{C}$.
Let $p \in] 0,1]$, a map $\|\|:. A \rightarrow[0, \infty[$ is a p-homogeneous seminorm if for $a, b$ in $\mathrm{A}$ and $\alpha$ in $\mathrm{K}$, $\|a+b\| \leq\|a\|+\|b\|$ and $\|\alpha a\|=|\alpha|^{p}\|a\|$. Moreover, if $\|a\|=0$ imply that $a=0$, $\|$.$\| is called a p-homo-$ geneous norm. A 1-homogeneous seminorm (resp.norm) is called a seminorm (resp.norm). $\|$.$\| is submultipli-$ cative if $\|a b\| \leq\|a\|\|b\|$ for all $a, b$ in $A$. $\|$.$\| has the$ square property if $\left\|a^{2}\right\|=\|a\|^{2}$ for all $a \in A$. If $\|$.$\| is a$ submultiplicative $\mathrm{p}$-homogeneous norm on $A$, then $(A,\|\|$.$) is called a p-normed algebra, we denote by \mathrm{M}(A)$ the set of all nonzero continuous multiplicative linear functionals on $A$. A complete p-normed algebra is called a p-Banach algebra. A uniform p-normed algebra is a p-normed algebra $(A,\|\|$.$) such that \left\|a^{2}\right\|=\|a\|^{2}$ for all $a \in A$. Let $A$ be a complex algebra with unit $e$, the spectrum of an element $a \in A$ is defined by

$$
\operatorname{Sp}(a)=\left\{\alpha \in C, \alpha e-a \notin A^{-1}\right\}
$$

where $A^{-1}$ is the set of all invertible elements of $A$. Let $A$ be a real algebra with unit $e$, the spectrum of $a \in A$ is defined by

$$
S p(a)=\left\{s+i t \in C,(a-s e)^{2}+t^{2} e \notin A^{-1}\right\} .
$$

Let $A$ be an algebra, the spectral radius of an element $a \in A$ is defined by $r(a)=\sup \{|\alpha|, \alpha \in \operatorname{Sp}(a)\}$. Let $(A,\|\|$.$) be a p-normed algebra, the \operatorname{limit} \lim _{n \rightarrow \infty}\left\|a^{n}\right\|^{\frac{1}{p n}}$ exists for each $a \in A$, and if $\mathrm{A}$ is complete, we have 
$r(a)=\lim _{n \rightarrow \infty}\left\|a^{n}\right\|^{\frac{1}{p n}}$ for all $a \in A$. A $*$-algebra is a complex algebra with a mapping $*: A \rightarrow A, a \rightarrow a^{*}$, such that, for $a, b$ in A and $\alpha \in C$,

$$
\begin{aligned}
& \left(a^{*}\right)^{*}=a,(a+b)^{*}=a^{*}+b^{*}, \\
& (\alpha a)^{*}=\bar{\alpha} a^{*},(a b)^{*}=b^{*} a^{*} .
\end{aligned}
$$

The map * is called an involution on A. An element $a \in A$ is said to be hermitian if $a^{*}=a$. The set of all hermitian elements of $\mathrm{A}$ is denoted by $\mathrm{H}(A)$.

\section{A Functional Representation Theorem for a Class of Real p-Banach Algebras}

We will need the following result due to B. Aupetit and J. Zemanek ([9,10]), their algebraic approach works for real p-Banach algebras.

Theorem 3.1. Let $A$ be a real p-Banach algebra with unit. If there is a positive constant $\alpha$ such that $r(a b) \leq \alpha r(a) r(b)$ for all $a, b$ in $A$, then for every irreducible representation $\pi$ of $A$ on a real linear space $E$, the algebra $\pi(A)$ is isomorphic (algebraically) to its commutant in the algebra $L(E)$ of all linear transformations on $E$.

Let $A$ be a real p-Banach algebra with unit such that $\|a\|^{\frac{1}{p}} \leq m r(a)$ for some positive constant $m$ and all $a \in A$. Let $X(A)$ be the set of all nonzero multiplicative linear functionals from $A$ into the noncommutative algebra $H$ of quaternions. For $a \in A$, we consider the map $J(a): X(A) \rightarrow H, J(a) x=x(a)$ for all $x \in X(A)$. We endow $X(A)$ with the weakest topology such that all the functions $J(a), a \in A$, are continuous. The map $J: A \rightarrow C(X(A), H), a \rightarrow J(a)$, is a homomorphism from $A$ into the real algebra of all continuous functions from $X(A)$ into $H$.

Theorem 3.2. If $\pi$ is an irreducible representation of $A$, then $\pi(A)$ is isomorphic to $R, C$ or $H$.

Proof. Let $a, b \in A$ and $n \geq 1$, we have

$$
\left\|(a b)^{n}\right\| \leq\|a\|^{n}\|b\|^{n}
$$

then

$$
\left\|(a b)^{n}\right\|^{\frac{1}{p n}} \leq\|a\|^{\frac{1}{p}}\|b\|^{\frac{1}{p}} .
$$

Letting $n \rightarrow \infty$, we obtain $r(a b) \leq m^{2} r(a) r(b)$. Let $\pi$ be an irreducible representation of $A$ on a real linear space $E$. By Theorem 3.1, $\pi(A)$ is isomorphic to its commutant $\mathrm{Q}$ in the algebra $L(E)$ of all linear transformations on $E$. Let $y_{0}$ be a fixed nonzero element in $E$. For $y \in E$, we consider

$$
\|y\|_{E}=\inf \left\{\|a\|, a \in A \text { and } \pi(a) y_{0}=y\right\} .
$$

By the same proof as in [11, Lemma 6.5], $\|\cdot\|_{E}$ is a p-norm on $E$ and $\mathrm{Q}$ is a real division p-normed algebra of continuous linear operators on $E$. By [12], Q is isomorphic to $R, C$ or $H$.

Proposition 3.3. $A$ is semisimple and $X(A)$ is a nonempty set which separates the elements of $A$.

Proof. By the condition $\|a\|^{\frac{1}{p}} \leq m r(a)$ for all $a \in A$, we deduce that $A$ is semisimple. Let $a$ be a nonzero element in $A$, since $A$ is semisimple, there is an irreducible representation $\pi$ of $A$ such that $\pi(a) \neq 0$. By Theorem 3.2, there is $\varphi: \pi(A) \rightarrow H$ an isomorphism (into). We consider the map $T=\varphi \circ \pi, T: A \rightarrow H$ is a multiplicative linear functional. Moreover,

$$
T(a)=\varphi(\pi(a)) \neq 0
$$

since $\pi(a) \neq 0$ and $\varphi$ is injective.

\section{Proposition 3.4.}

1) $|x(a)| \leq\|a\|^{\frac{1}{p}}$ for all $a \in A$ and $x \in X(A)$;

2) An element $a$ is invertible in $A$ if and only if $J(a)$ is invertible in $C(X(A), H)$;

3) $S p(a)=S p(J(a))$ for all $a \in A$.

Proof. (1): Since $H$ is a real uniform Banach algebra under the usual norm

$$
|.|,|x(a)|=r_{H}(x(a)) \leq r_{A}(a) \leq\|a\|^{\frac{1}{p}}
$$

for all $a \in A$ and $x \in X(A)$.

(2): The direct implication is obvious. Conversely, let $\pi$ be an irreducible representation of $A$. By Theorem 3.2, there is $\varphi: \pi(A) \rightarrow H$ an isomorphism (into). Since $\varphi \circ \pi \in X(A)$ and $J(a)$ is invertible,

$$
0 \neq J(a)(\varphi \circ \pi)=\varphi(\pi(a)),
$$

then $\pi(a) \neq 0$. Consequently, a is invertible.

(3): $s+i t \in \operatorname{Sp}(a)$ iff $(a-s e)^{2}+t^{2} e \notin A^{-1}$

Iff $J\left((a-s e)^{2}+t^{2} e\right) \notin C(X(A), H)^{-1}$ by (2)

Iff $(J(a)-s J(e))^{2}+t^{2} J(e) \notin C(X(A), H)^{-1}$

Iff $s+i t \in \operatorname{Sp}(J(a))$.

Proposition 3.5. $X(A)$ is a Hausdorff compact space.

Proof. Let $x_{1}, x_{2}$ in $X(A), x_{1} \neq x_{2}$, there is an element $a \in A$ such that $x_{1}(a) \neq x_{2}(a)$, i.e. $J(a) x_{1} \neq J(a) x_{2}$, so $X(A)$ is Hausdorff. Let $a \in A$ and

$$
K_{a}=\left\{q \in H,|q| \leq\|a\| \frac{1}{p}\right\},
$$

$K_{a}$ is compact in $H$. Let $K$ be the topological product of $K_{a}$ for all $a \in A, K$ is compact by the Tychonoff Theorem. By Proposition 3.4(1), $X(A)$ is a subset of $K$. It is easy to see that the topology of 
$X(A)$ is the relative topology from $K$ and that $X(A)$ is closed in $K$. Then $X(A)$ is compact.

Theorem 3.6. The map

$$
J: A \rightarrow C(X(A), H), a \rightarrow J(a),
$$

is an isomorphism (into) such that

$$
m^{-1}\|a\|^{\frac{1}{p}} \leq\|J(a)\|_{s} \leq\|a\|^{\frac{1}{p}}
$$

for all $a \in A$, where \|\|$_{s}$ is the supnorm on $C(X(A), H)$. If $m=1$, we have $\|a\|^{\frac{1}{p}}=\|J(a)\|_{s}$ for all $a \in A$.

Proof. By Proposition 3.3, $J$ is an injective homomorphism. Let $a \in A$, by Proposition 3.4(3),

$$
r(a)=r(J(a))=\|J(a)\|_{s}
$$

since $C(X(A), H)$ is a real uniform Banach algebra under the supnorm $\|\cdot\|_{s}$. Moreover, $\|J(a)\|_{s} \leq\|a\|^{\frac{1}{p}}$ by Proposition 3.4(1). Then

$$
m^{-1}\|a\|^{\frac{1}{p}} \leq r(a)=\|J(a)\|_{s} \leq\|a\|^{\frac{1}{p}} .
$$

As an application, we obtain an extension of the Kulkarni Theorem [13, Theorem 1] to real p-Banach algebras.

Theorem 3.7. Let $a$ be an element in $A$ such that $S p(a) \subset R$, then $a$ belongs to the center of $A$.

Proof. By Theorem 3.6, $J: A \rightarrow C(X(A), H)$ is an isomorphism (into). Let $a \in A$ with $S p(a) \subset R$. Let $x \in X(A)$ and $x(a)=s+t$ where $s \in R$ and

$$
t=t_{1} i+t_{2} j+t_{3} k
$$

Suppose that $t \neq 0$. We have

$$
(x(a)-s)^{2}=t^{2}=-\left(t_{1}^{2}+t_{2}^{2}+t_{3}^{2}\right)=-|t|^{2},
$$

Then

$$
(x(a)-s)^{2}+|t|^{2}=0 .
$$

Consequently

$$
s+i|t| \in S p(x(a)) \subset S p(a)
$$

with $|t| \neq 0$, a contradiction. Then

$$
J(a) \in C(X(A), R)
$$

and

$$
J(a) J(b)=J(b) J(a)
$$

for all $b$ in $A$, i.e. $J(a b-b a)=0$ for all $b$ in $A$. Since $J$ is injective, $a b-b a=0$ for all $b$ in $A$.

\section{A Functional Representation Theorem for a Class of Complex p-Banach Algebras}

Let $\|\cdot\|$ be a submultiplicative p-homogeneous se- minorm on a complex algebra $A$. For $a \in A,|a|$ is defined as follows:

$$
|a|=\inf \sum_{i=1}^{n}\left\|a_{i}\right\|^{\frac{1}{p}},
$$

where the infimum is taken over all decompositions of $a$ satisfying the condition $a=\sum_{i=1}^{n} a_{i}, a_{1}, \cdots, a_{n} \in A$. By [14, Theorem 1], .. is a submultiplicative seminorm on $A$, it is called the support seminorm of $\|$.$\| . Also, it$ is shown [14] the following result:

Theorem 2 of [14]. Let $A$ be a complex algebra, \|.. a submultiplicative p-homogeneous seminorm on $A$, and $|$.$| the support seminorm of \|$.$\| . Then$

$$
\lim _{n \rightarrow \infty}\left\|a^{n}\right\| \frac{1}{p n}=\lim _{n \rightarrow \infty}\left|a^{n}\right|^{\frac{1}{n}}
$$

for all $a \in A$.

In the proof of this theorem, Xia Dao-Xing uses the following inequality: If $a=a_{1}+\cdots+a_{m}$ and $n \geq 1$, then

$$
\left\|a^{n}\right\| \leq \sum_{\alpha_{1}+\cdots+\alpha_{m}=n}\left(\frac{n !}{\alpha_{1} ! \cdots \alpha_{m} !}\right)^{p}\left\|a_{1}\right\|^{\alpha_{1}} \cdots\left\|a_{m}\right\|^{\alpha_{m}} .
$$

If the algebra is commutative,

$$
\begin{aligned}
a^{n} & =\left(a_{1}+\cdots+a_{m}\right)^{n} \\
& =\sum_{\alpha_{1}+\cdots+\alpha_{m}=n} \frac{n !}{\alpha_{1} ! \cdots \alpha_{m} !} a_{1}^{\alpha_{1}} \cdots a_{m}^{\alpha_{m}},
\end{aligned}
$$

then

$$
\left\|a^{n}\right\| \leq \sum_{\alpha_{1}+\cdots+\alpha_{m}=n}\left(\frac{n !}{\alpha_{1} ! \cdots \alpha_{m} !}\right)^{p}\left\|a_{1}\right\|^{\alpha_{1}} \cdots\left\|a_{m}\right\|^{\alpha_{m}} .
$$

This inequality is not justified in the noncommutative case; if the algebra is noncommutative, we only have

$$
\left\|a^{n}\right\| \leq \sum_{\alpha_{1}+\cdots+\alpha_{m}=n} \frac{n !}{\alpha_{1} ! \cdots \alpha_{m} !}\left\|a_{1}\right\|^{\alpha_{1}} \cdots\left\|a_{m}\right\|^{\alpha_{m}} .
$$

For the sequel, we will use Theorem 2 of [14] in the commutative case.

Theorem 4.1. Let $(A,\|\|$.$) be a complex p-normed$ algebra such that $\|a\|^{2} \leq m\left\|a^{2}\right\|$ for some positive constant $m$ and all $a \in A$. Then $|a| \leq\|a\|^{\frac{1}{p}} \leq m^{\frac{1}{p}}|a|$ and $|a|^{2} \leq m^{\frac{2}{p}}\left|a^{2}\right|$ for all $a \in A$, where $\|$ is the support seminorm of $\|\cdot\|$.

Proof. The completion $B$ of $(A,\|\|$.$) is a p-Banach$ algebra such that $\|b\|^{2} \leq m\left\|b^{2}\right\|$ for all $b \in B$, it is commutative by [1, Lemma 1$]$, so $A$ is commutative. By induction, $\|a\| \leq m^{1-2^{-n}}\left\|a^{2^{n}}\right\|^{2^{-n}}$ for all $a \in A$ and $n \geq 1$, then $\|a\| \leq m \lim _{n \rightarrow \infty}\left\|a^{n}\right\|^{\frac{1}{n}}$ for all $a \in A$. By the commutative version of [14, Theorem 2], we have 


$$
\begin{aligned}
|a| & \leq\|a\|^{\frac{1}{p}} \leq m^{\frac{1}{p}} \lim _{n \rightarrow \infty}\left\|a^{n}\right\| \frac{1}{p^{n}} \\
& =m^{\frac{1}{p}} \lim _{n \rightarrow \infty}\left|a^{n}\right|^{\frac{1}{n}} \leq m^{\frac{1}{p}}|a|
\end{aligned}
$$

for all $a \in A$. From the above inequalities,

$$
|a|^{2} \leq\|a\|^{\frac{2}{p}} \leq\left(m\left\|a^{2}\right\|\right)^{\frac{1}{p}} \leq m^{\frac{2}{p}}\left|a^{2}\right| .
$$

Corollary 4.2. Let $(A,\|\|$.$) be a complex uniform$ p-normed algebra. Then $|a|=\|a\|^{\frac{1}{p}}$ for all $a \in A$.

Theorem 4.3. Let $(A,\|\|$.$) be a complex p-Banach$ algebra with unit such that $\|a\|^{2} \leq m\left\|a^{2}\right\|$ for some positive constant $m$ and all $a \in A$. Then the Gelfand map $G: A \rightarrow C(M(A))$ is an isomorphism (into) such that

$$
m^{-\frac{2}{p}}\|a\|^{\frac{1}{p}} \leq m^{-\frac{1}{p}}|a| \leq\|G(a)\|_{s} \leq|a| \leq\|a\|^{\frac{1}{p}}
$$

for all $a \in A$, where \|\|$_{S}$ is the supnorm on $C(M(A))$.

Proof. A is commutative by [1, Lemma 1]. By Theorem

4.1, $|a| \leq\|a\|^{\frac{1}{p}} \leq m^{\frac{1}{p}}|a|$ for all $a \in A$, then $(A,|\cdot|)$ is a complex commutative Banach algebra with unit. Clearly $M(A)=M(A,\|\cdot\|)=M(A,||$.$) is a nonempty compact$ space. As in the proof of Theorem 4.1, we have

$$
\begin{aligned}
|a| & \leq m^{\frac{1}{p}} \lim _{n \rightarrow \infty}\left|a^{n}\right|^{\frac{1}{n}} \\
& =m^{\frac{1}{p}} \sup \{|f(a)|, f \in M(A)\} \\
& =m^{\frac{1}{p}}\|G(a)\|_{s} \leq m^{\frac{1}{p}}|a| .
\end{aligned}
$$

Let $a \in A$, from the above inequalities,

$$
m^{-\frac{2}{p}}\|a\|^{\frac{1}{p}} \leq m^{-\frac{1}{p}}|a| \leq\|G(a)\|_{s} \leq|a| \leq\|a\|^{\frac{1}{p}} .
$$

Corollary 4.4. Let $(A,\|\|$.$) be a complex uniform$ p-Banach algebra with unit. Then the Gelfand map $G: A \rightarrow C(M(A))$ is an isomorphism (into) such that

$$
|a|=\|a\| \frac{1}{p}=\|G(a)\|_{s}
$$

for all $a \in A$.

Theorem 4.5. Let $(A,\|\|$.$) be a complex p-normed$ * -algebra with unit such that

1) $\|a\|^{2} \leq m\left\|a^{2}\right\|$ for some positive constant $m$ and all $a \in A$;

2) Every element in $H(A)$ has a real spectrum in the completion $B$ of $A$.

Then the involution $*$ is continuous on $A$ and the Gelfand map $G: B \rightarrow C(M(B))$ is a $*$-isomorphism such that $m^{-\frac{2}{p}}\|b\|^{\frac{1}{p}} \leq\|G(b)\|_{S} \leq\|b\|^{\frac{1}{p}}$ for all $b$ in $B$.
Proof. By Theorem 4.3, it remains to show that the involution * is continuous on $A, G\left(b^{*}\right)=G(b)^{*}$ for all $b \in B$, and $G$ is surjective. Let $h \in H(A)$,

$$
S p_{B}(h)=\{f(h), f \in M(B)\} \subset R
$$

by (2). Let $a \in A$, we have $a=h_{1}+i h_{2}$ with $h_{1}, h_{2}$ $\in H(A)$. Let $f \in M(B)$,

$$
\begin{aligned}
f\left(a^{*}\right) & =f\left(h_{1}-i h_{2}\right)=f\left(h_{1}\right)-i f\left(h_{2}\right) \\
& =\left(f\left(h_{1}\right)+i f\left(h_{2}\right)\right)^{*}=f\left(h_{1}+i h_{2}\right)^{*}=f(a)^{*}
\end{aligned}
$$

since $f\left(h_{1}\right)$ and $f\left(h_{2}\right)$ are real. Then $G\left(a^{*}\right)=G(a)^{*}$ for all $a \in A$. By Theorem 4.3,

$$
\begin{aligned}
& m^{-\frac{2}{p}}\left\|a^{*}\right\|^{\frac{1}{p}} \leq\left\|G\left(a^{*}\right)\right\|_{S} \\
& =\left\|G(a)^{*}\right\|_{S}=\|G(a)\|_{S} \leq\|a\| \frac{1}{p}
\end{aligned}
$$

for all $a \in A$, then $\left\|a^{*}\right\| \leq m^{2}\|a\|$ for all $a \in A$. Consequently, the involution $*$ is continuous on $\mathrm{A}$ and can be extended to a continuous involution on $B$ which we will also denote by $*$. Let $b \in B$, there exists a sequence $\left(a_{n}\right)_{n}$ in $A$ such that $a_{n} \rightarrow b$. Since the involution on $B$ and the Gelfand map $G: B \rightarrow C(M(B))$ are continuous, we have

$$
G\left(a_{n}^{*}\right) \rightarrow G\left(b^{*}\right)
$$

and

$$
G\left(a_{n}\right)^{*} \rightarrow G(b)^{*}
$$

then

$$
G\left(b^{*}\right)=G(b)^{*} \text {. }
$$

By the Stone-Weierstrass Theorem, we deduce that $G$ is surjective.

As a consequence, we obtain the main result in [8].

Corollary 4.6. Let $A$ be a complex uniform pnormed *-algebra with unit such that every element in $H(A)$ has a real spectrum in the completion $B$ of $A$. then $B$ is a commutative $C^{*}$-algebra.

\section{The Main Result}

Theorem 5.1. Let $A$ be a real associative algebra. Every p-homogeneous seminorm $q$ with square property on $A$ is submultiplicative and $q^{\frac{1}{p}}$ is a submultiplicative seminorm on $A$.

Proof. By [1], there exists a positive constant $m$ such that $q(a b) \leq m q(a) q(b)$ for all $a, b \in A$. $\operatorname{Ker}(q)$ is an ideal of $A$, the norm $|$.$| on the quotient algebra$ $A / \operatorname{Ker}(q)$ defined by $|a+\operatorname{Ker}(q)|=q(a)$ is a p-norm with square property. Define

$$
\|a+\operatorname{Ker}(q)\|=m|a+\operatorname{Ker}(q)|
$$

for all $a \in A$. Let $a, b \in A$, 


$$
\begin{aligned}
\|a b+\operatorname{Ker}(q)\| & =m|a b+\operatorname{Ker}(q)| \\
& \leq m^{2}|a+\operatorname{Ker}(q) \| b+\operatorname{Ker}(q)| \\
& =\|a+\operatorname{Ker}(q)\|\|b+\operatorname{Ker}(q)\|,
\end{aligned}
$$

then $(A / \operatorname{Ker}(q),\|\|$.$) is a real p-normed algebra. Let$ $a \in A$,

$$
\begin{aligned}
\left\|a^{2}+\operatorname{Ker}(q)\right\| & =m\left|a^{2}+\operatorname{Ker}(q)\right| \\
& =m|a+\operatorname{Ker}(q)|^{2} \\
& =\left.m^{-1}(m \mid a+\operatorname{Ker}(q))\right|^{2} \\
& =m^{-1} \mid a+\operatorname{Ker}(q) \|^{2}
\end{aligned}
$$

i.e.

$$
\|a+\operatorname{Ker}(q)\|^{2}=m\left\|a^{2}+\operatorname{Ker}(q)\right\| .
$$

The completion $B$ of $(A / \operatorname{Ker}(q),\|\|$.$) satisfies also$ the property $\|b\|^{2}=m\left\|b^{2}\right\|$ for all $b \in B$, and by induction $\|b\|=m^{1-2^{-n}}\left\|b^{2^{n}}\right\|^{2^{-n}}$ for all $b \in B$ and $n \geq 1$, then $\|b\|=m r(b)^{p}$ for all $b \in B$. We consider two cases:

$B$ is unital: By section $3, X(B)$ is a nonempty compact space and the map $J: B \rightarrow C(X(B), H)$ is an isomorphism (into). By Proposition 3.4(3), $r(b)=r(J(b))$ for all $b \in B$. Let $b \in B$,

$$
\|b\|=m r(b)^{p}=m r(J(b))^{p}=m\|J(b)\|_{s}^{p}
$$

since $C(X(B), H)$ is a real uniform Banach algebra under the supnorm $\|\cdot\|_{s}$. Then $|b|=m^{-1}\|b\|=\|J(b)\|_{s}^{p}$ for all $b \in A / \operatorname{Ker}(q)$, so $\|$ is submultiplicative and $|\cdot|^{\frac{1}{p}}$ is a submultiplicative norm. Consequently, $q$ is submultiplicative and $q^{\frac{1}{p}}$ is a submultiplicative seminorm.

$B$ is not unital: Let $B_{1}$ be the algebra obtained from $B$ by adjoining the unit. By the same proof of [15, Lemma 2] which works for real p-Banach algebras, there exists a p-norm $N$ on $B_{1}$ such that

1) $\left(B_{1}, N\right)$ is a real p-Banach algebra with unit;

2) $N(b)^{\frac{1}{p}} \leq m^{3} r_{B_{1}}(b)$ for all $b \in B_{1}$;

3) $N$ and $\|$.$\| are equivalent on B$.

By section $3, X\left(B_{1}\right)$ is a nonempty compact space and the map $J: B_{1} \rightarrow C\left(X\left(B_{1}\right), H\right)$ is an isomorphism (into). Let $b \in B$,

$$
\|b\|=m r_{B}(b)^{p}=m r_{B_{1}}(b)^{p}
$$

by (3) $=m r(J(b))^{p}$ by Proposition 3.4(3)

$=m\|J(b)\|_{S}^{p}$ by the square property of the supnorm.

Then $|b|=m^{-1}\|b\|=\|J(b)\|_{s}^{p}$ for all $b \in A / \operatorname{Ker}(q)$, so $\|$ is submultiplicative and $\mid \cdot \frac{1}{p}$ is a submultiplicative norm. Consequently, $q$ is submultiplicative and $q^{\frac{1}{p}}$ is a submultiplicative seminorm.

\section{REFERENCES}

[1] J. Arhippainen, "On Locally Pseudoconvex Square Algebras,” Publicacions Matematiques, Vol. 39, No. 1, 1995, pp. 89-93. http://dx.doi.org/10.5565/PUBLMAT_39195_06

[2] S. J. Bhatt and D. J. Karia, "Uniqueness of the Uniform Norm with an Application to Topological Algebras," Proceedings of the American Mathematical Society, Vol. 116, 1992, pp. 499-504.

http://dx.doi.org/10.1090/S0002-9939-1992-1097335-4

[3] S. J. Bhatt, "A Seminorm with Square Property on a Banach Algebra Is Submultiplicative," Proceedings of the American Mathematical Society, Vol. 117, 1993, pp. 435438.

http://dx.doi.org/10.1090/S0002-9939-1993-1128724-8

[4] H. V. Dedania, "A Seminorm with Square Property Is Automatically Submultiplicative," Proceedings of the Indian Academy of Science, Vol. 108, 1998, pp. 51-53.

[5] Z. Sebestyen, "A Seminorm with Square Property on a Complex Associative Algebra Is Submultiplicative,” Proceedings of the American Mathematical Society, Vol. 130, 2001, pp. 1993-1996.

http://dx.doi.org/10.1090/S0002-9939-01-06278-5

[6] M. El Azhari, “A Real Seminorm with Square Property Is Submultiplicative,” Indian Journal of Pure and Applied Mathematics, Vol. 43, No. 4, 2012, pp. 303-307. http://dx.doi.org/10.1007/s13226-012-0018-z

[7] M. Abel and K. Jarosz, "Noncommutative Uniform Algebras,” Studia Mathematica, Vol. 162, 2004, pp. 213218. http://dx.doi.org/10.4064/sm162-3-2

[8] A. El Kinani, “On Uniform Hermitian p-Normed Algebras,” Turkish Journal of Mathematics, Vol. 30, 2006, pp. 221-231.

[9] B. Aupetit and J. Zemanek, "On the Real Spectral Radius in Real Banach Algebras,” Bulletin de l'Académie Polonaise des Sciences Série des Sciences Math., Astr. et Phys., Vol. 26, 1978, pp. 969-973.

[10] J. Zemanek, "Properties of the Spectral Radius in Banach Algebras,” Banach Center Publications, Vol. 8, 1982, pp. 579-595.

[11] A. M. Sinclair, "Automatic Continuity of Linear Operators,” Cambridge University Press, Cambridge, 1976. http://dx.doi.org/10.1017/CBO9780511662355

[12] Ph. Turpin, “Sur une Classe D’algèbres Topologiques,” C. R. Acad. Sc. Paris, Série A, Vol. 263, 1966, pp. 436-439. 
[13] S. H. Kulkarni, "Representations of a Class of Real $B^{*}$-Algebras as Algebras of Quaternion-Valued Functions," Proceedings of the American Mathematical Society, Vol. 116, 1992, pp. 61-66.

[14] D.-X. Xia, “On Locally Bounded Topological Algebras,” Acta Mathematica Sinica, Vol. 14, 1964, pp. 261-276.
[15] R. A. Hirschfeld and W. Zelazko, "On Spectral Norm Banach Algebras,” Bulletin de l'Académie Polonaise des Sciences Série des Sciences Math., Astr. et Phys., Vol. 16, 1968, pp. 195-199. 